\title{
Type 2 Autoimmune Pancreatitis (Idiopathic Duct-Centric Pancreatitis) Highlighting Patients Presenting as Clinical Acute Pancreatitis: A Single- Center Experience
}

\author{
Dongwook Oh ${ }^{1}$, Tae Jun Song ${ }^{1}$, Sung-Hoon Moon ${ }^{2}$, Jin Hee Kim ${ }^{3}$, Nam Joo Lee ${ }^{3}$, Seung-Mo Hong ${ }^{4}$, Joune Seup Lee ${ }^{1}$, \\ Seok Jung Jo ${ }^{1}$, Dong Hui Cho ${ }^{1}$, Do Hyun Park ${ }^{1}$, Sang Soo Lee ${ }^{1}$, Dong-Wan Seo ${ }^{1}$, Sung Koo Lee ${ }^{1}$, and Myung-Hwan Kim ${ }^{1}$ \\ ${ }^{1}$ Department of Gastroenterology, Asan Medical Center, University of Ulsan College of Medicine, Seoul, ${ }^{2}$ Department of Internal Medicine, \\ Hallym University Sacred Heart Hospital, Hallym University College of Medicine, Anyang, Departments of ${ }^{3}$ Radiology and ${ }^{4}$ Pathology, Asan \\ Medical Center, University of Ulsan College of Medicine, Seoul, Korea
}

See editorial on page 385.

Background/Aims: Type 2 autoimmune pancreatitis (AIP) has been considered extremely rare in East Asia. This study aimed to clarify the prevalence, clinical characteristics and radiological findings of type 2 AIP highlighting patients presenting as acute pancreatitis in a single center. Methods: Type 2 AIP patients were classified according to International Consensus Diagnostic Criteria. Radiological findings were compared between type 2 AIP presenting as acute pancreatitis and gallstone pancreatitis. Results: Among 244 patients with AIP, 27 (11.1\%) had type 2 AIP (definite, 15 [55.5\%] and probable 12 [44.5\%]). The median age of patients with type 2 AIP was 29 years (interquartile range, 20 to 39 years). Acute pancreatitis was the most common initial presentation ( $n=17,63 \%$ ) while obstructive jaundice was present in only one patient. Ulcerative colitis (UC) was associated with type 2 AIP in $44.4 \%(12 / 27)$ of patients. Radiological pancreatic imaging such as delayed enhancement of diffusely enlarged pancreas, homogeneous enhancement of focal enlargement/mass, absent/minimal peripancreatic fat infiltration or fluid collection, and multifocal main pancreatic duct narrowings were helpful for differentiating type 2 AIP from gallstone pancreatitis. During follow-up (median, 32.3 months), two patients (2/25, $8 \%)$ experienced relapse. Conclusions: In South Korea, type 2 AIP is not as rare as previously thought. Overall, the clinical profile of type 2 AIP was similar to that of Western countries. Type 2 AIP should be considered in young
UC patients with acute pancreatitis of uncertain etiology. (Gut Liver 2019;13:461-470)

Key Words: Autoimmune pancreatitis; Idiopathic duct-centric pancreatitis; Acute pancreatitis

\section{INTRODUCTION}

With improved understanding of autoimmune pancreatitis (AIP) and its clinical profiles, two histological subtypes have been recognized; type 1 (also referred to as lymphoplasmacytic sclerosing pancreatitis) and type 2 (also referred to as idiopathic duct-centric pancreatitis [IDCP]). ${ }^{1}$ In contrast to type 1 AIP that is viewed as the pancreatic manifestation of immunoglobulin G4 (IgG4)-related systemic fibroinflammatory disease, type 2 AIP is a pancreas-specific disorder not associated with IgG4. ${ }^{2}$

While the knowledge of the type 1 AIP has advanced significantly over the past decade, the understanding of type 2 AIP has been slower and less is known about type 2 AIP. ${ }^{3}$ In a study by Kamisawa et al., ${ }^{4}$ patients with type 2 AIP were only two (1.2\%) among 165 Japanese AIP patients and seven (5.6\%) among 124 Korean patients. It was thought that AIP in East Asia was exclusively type 1 AIP. ${ }^{4}$ Type 2 AIP is, however, increasingly being recognized in South Korea and Japan, ${ }^{5,6}$ raising the possibility that the disease was overlooked for many years and was referred to by different names. Type 2 AIP may be mislabeled as simply AIP (without subtype specification), or AIP-NOS (not otherwise specified). Also, type 2 AIP presenting as clinical acute pancreatitis can be mistakenly classified as id-

\footnotetext{
Correspondence to: Myung-Hwan Kim

Department of Gastroenterology, Asan Medical Center, University of Ulsan College of Medicine, 88 Olympic-ro 43-gil, Songpa-gu, Seoul 05505, Korea

Tel: +82-2-3010-3183, Fax: +82-2-476-0824, E-mail: mhkim@amc.seoul.kr

Received on September 20, 2018. Revised on November 4, 2018. Accepted on November 10, 2018. Published online April 17, 2019

pISSN 1976-2283 eISSN 2005-1212 https://doi.org/10.5009/gnl18429

Dongwook Oh and Tae Jun Song contributed equally to this work as first authors.

(a) This is an Open Access article distributed under the terms of the Creative Commons Attribution Non-Commercial License (http://creativecommons.org/licenses/by-nc/4.0) which permits unrestricted non-commercial use, distribution, and reproduction in any medium, provided the original work is properly cited.
} 
iopathic acute pancreatitis, if the cause is unrecognized. Since a pancreatic histology is needed for a definitive diagnosis of type 2 AIP, under-recognition of type 2 AIP is more likely than that of type 1 AIP.?

In 2011, the International Consensus Diagnostic Criteria (ICDC) for AIP were published by the International Association of Pancreatology. ${ }^{8}$ According to the ICDC, AIP was subtyped as type 1 and type 2 , and each was subdivided into definite and probable diagnosis. In the present study, type 2 AIP patients were classified according to the ICDC. Until now, only a few articles from East Asia have addressed the issues about type 2 AIP.

We aimed to clarify the prevalence, clinical characteristics, and radiological features of type 2 AIP highlighting patients presenting as clinical acute pancreatitis in our cohorts and compare our results with those from Western countries. We also compared radiological findings between type 2 AIP manifesting as acute pancreatitis and acute interstitial edematous pancreatitis of gallstone etiology.

\section{MATERIALS AND METHODS}

From January 2003 to December 2016, adult AIP patients $(\geq 18$ years old) were enrolled from our prospectively collected database of AIP cohorts. Patients met the Asian diagnostic criteria or the HISORt criteria for AIP until 2011, and the ICDC thereafter. $^{8-10}$ Some of our study cohort involve previously reported cases $^{5}$ and pathology slides were re-reviewed by an experienced pathologist (S.M.H.) specialized in AIP.

Patients with type 2 AIP were reassessed clinically, radiologically, and histologically, and classified as having "definite" or "probable" according to the ICDC (Table 1). ${ }^{8}$ A definitive diagnosis of type 2 AIP can be made when granulocytic epithelial lesion (GEL) is demonstrated on pancreatic histology. ${ }^{3}$ Definitive type 2 AIP can also be diagnosed even if GEL is not observed if patients have level 2 histological findings on pancreatic core biopsy (PCB), inflammatory bowel disease (IBD), and steroid responsiveness. On the other hand, in patients with IBD, probable type 2 AIP can be established without histology in the presence of steroid responsiveness of pancreatic abnormalities. In the absence of IBD, probable type 2 AIP can be diagnosed when patients have level 2 histological findings on PCB and steroid responsiveness.

At the beginning of this study, percutaneous transabdominal ultrasound (US)-guided PCB was commonly performed with 18-gauge needles (Stericut 18G coaxial; TSK Laboratory, Tochigi, Japan). After the introduction of endoscopic ultrasoundguided fine pancreatic core biopsy (EUS-PCB) at our institute, EUS-PCB was performed with 19-gauge TruCut needles (QuickCore; Wilson-Cook, Winston-Salem, NC, USA). Since TruCut needles were not available in practice, 19- or 22-gauge ProCore needles (EchoTip Procore; Cook Medical, Bloomington, IN, USA) were used.

Clinical acute pancreatitis was defined as characteristic abdominal pain necessitating parenteral analgesics including opioids and elevated serum pancreatic enzyme level (amylase and/ or lipase) greater than 3 times the upper normal limits. ${ }^{11,12}$ Painless obstructive jaundice was defined as a serum total bilirubin level of $>3.0 \mathrm{mg} / \mathrm{dL}$ associated with abnormal liver chemistry and bile duct dilatation. Written informed consent to participate in this study was provided by all patients. This study was approved by the Institutional Review Board of Asan Medical Center (IRB number: 2015-0865).

\section{Evaluation of radiological findings}

To elucidate the differences of radiological findings, ordinary acute interstitial edematous pancreatitis due to gallstones was compared with type 2 AIP presenting as clinical acute pancreatitis because gallstone is one of the most common causes of acute pancreatitis. Control subjects who underwent computed tomography (CT) and/or magnetic resonance imaging (MRI) from September 2016 to November 2016 were consecutively selected among patients with acute interstitial edematous pancreatitis caused by gallstones from our institutional imaging database. Necrotizing pancreatitis of gallstone etiology was

Table 1. International Consensus Diagnostic Criteria for the Diagnosis of Type 2 Autoimmune Pancreatitis

\begin{tabular}{ccc}
\hline Diagnosis & Imaging evidence & Collateral evidence \\
Definitive type 2 AIP & Histologically confirmed IDCP (level 1H) \\
Subgroup 1 & Typical/indeterminate & Clinical IBD + level 2H + Rt \\
Subgroup 2 & Typical/indeterminate & Clinical IBD + Rt \\
Probable type 2 AIP & & Level 2H + Rt \\
Subgroup 1 & Typical/indeterminate & Typical/indeterminate \\
Subgroup 2 & &
\end{tabular}

AIP, autoimmune pancreatitis; IDCP, idiopathic duct-centric pancreatitis; H, histology of pancreas; IBD, inflammatory bowel disease; Rt, steroid responsiveness; IgG4, immunoglobulin G4.

Level $1 \mathrm{H},(1)$ granulocytic infiltration of duct wall with or without granulocytic acinar inflammation and (2) absent or scant (0-10 cells/HPF) IgG4-positive cells; Level 2H, (1) granulocytic and lymphoplasmacytic acinar infiltrate and (2) absent or scant (0-10 cells/HPF) IgG4-positive cells. 
excluded because pancreatic and/or peripancreatic necrosis has rarely been described in AIP.

In the comparison of CT findings between type 2 AIP presenting as acute pancreatitis and ordinary acute interstitial edematous pancreatitis induced by gallstones, evaluation items were categorized as pancreas swelling, focal mass, peripancreatic halo, and delayed enhancement. The extent of pancreas swelling or enlargement was defined as follows: (1) diffuse (involved segment greater than half of the entire pancreas); (2) focal/segmental (involved segment less than half of the entire pancreas); and (3) multifocal (involved multifocal segment with intervening normal-looking pancreas). ${ }^{9,13}$ Focal mass was defined as an obvious hypoenhancing lesion on the arterial phase of contrastenhanced CT/MRI compared with the surrounding pancreatic tissue. ${ }^{5,9}$ Peripancreatic halo was defined as capsule-like rim of low-attenuation soft tissue surrounding the pancreas. ${ }^{14}$ Delayed enhancement was defined as an increase of $\geq 15$ Hounsfield units from the arterial phase to the portal venous phase. ${ }^{15}$ Peripancreatic fat infiltration was defined when peripancreatic fat planes were blurred and showed increased attenuation (also called fat stranding). Focal/segmental main pancreatic duct (MPD) dilatation was defined if the main duct measures greater than $3 \mathrm{~mm}$ in the head and $2 \mathrm{~mm}$ in the body/tail of the pan- creas. $^{16}$

In the comparison of magnetic resonance cholangiopancreatography (MRCP) findings, MPD stricture was categorized according to extent and multiplicity as follows: MPD narrowing, when the stricture involved any portion of the MPD regardless of the length; and multifocal type, when the stricture involved $\geq 2$ sites with intervening normal-looking MPD. ${ }^{17}$ Common bile duct (CBD) narrowing was also compared between patients with type 2 AIP and ordinary acute interstitial edematous pancreatitis. ${ }^{5}$ MRCP was performed at $1.5 \mathrm{~T}$ (Magnetom Avanto; Siemens Medical Systems, Erlangen, Germany) without secretin stimulation.

\section{Treatment strategy and response to steroids}

Steroid responsiveness was defined as complete resolution or marked improvement of pancreatic imaging findings (parenchyma and MPD) after steroid treatment. ${ }^{18}$ Relapse was defined as a reappearance of abnormal pancreatic imaging with or without a related AIP event (pancreatic pain, acute pancreatitis or obstructive jaundice). When such events occurred, relapse was documented using radiological imaging to assess recurrent pancreatic inflammation. $^{19}$

The typical induction dosage of oral prednisolone was 30-40

Table 2. Baseline Characteristics and Clinical Outcomes of All Patients with Type 2 AIP and Patients with Type 2 AIP Presenting as Clinical Acute Pancreatitis

\begin{tabular}{|c|c|c|}
\hline Characteristic & $\begin{array}{c}\text { Overall type } 2 \text { AIP } \\
(\mathrm{n}=27)\end{array}$ & $\begin{array}{l}\text { Type } 2 \text { AIP presenting as } \\
\text { acute pancreatitis }(\mathrm{n}=17)\end{array}$ \\
\hline Age, yr & $29(20-39)$ & $29(21-38)$ \\
\hline Sex, male:female & 19:8 & $12: 5$ \\
\hline \multicolumn{3}{|l|}{ Initial symptom \& sign } \\
\hline Clinical acute pancreatitis & $17(63)$ & $17(100)$ \\
\hline Abdominal pain without biochemical evidence for acute pancreatitis & $6(22.2)$ & None \\
\hline Diarrhea and/or abdominal discomfort & $2(7.4)$ & None \\
\hline Painless obstructive jaundice & $1(3.7)$ & None \\
\hline Abnormal liver biochemistry without jaundice & $1(3.7)$ & None \\
\hline Recurrent pancreatitis & $9(33.3)$ & $9(52.9)$ \\
\hline Patients visiting the emergency department & $12(44.4)$ & 12 (70.6) \\
\hline \multicolumn{3}{|l|}{ Serology (IgG4) } \\
\hline IgG4, 1-2×ULN (135-270 mg/dL) & $2(7.4)$ & $1(5.9)$ \\
\hline IgG4, >2 ×ULN (>270 mg/dL) & 0 & 0 \\
\hline Ulcerative colitis & $12(44.4)$ & $8(47.1)$ \\
\hline Patients who underwent tissue acquisition & $20(74.1)$ & - \\
\hline EUS-guided pancreatic core biopsy & $16(59.3)$ & $10(58.8)$ \\
\hline Percutaneous transabdominal ultrasound-guided core biopsy & $3(11.1)$ & $1(5.9)$ \\
\hline Surgical resection & $1(3.7)$ & $1(5.9)$ \\
\hline Definite type 2 AIP & $15(55.5)$ & $5(29.4)$ \\
\hline Probable type 2 AIP & $12(44.5)$ & $12(76.5)$ \\
\hline
\end{tabular}

Data are presented as median (interquartile range) or number (\%).

AIP, autoimmune pancreatitis; IgG4, immunoglobulin G4; ULN, upper limit of normal; EUS, endoscopic ultrasound. 
$\mathrm{mg} /$ day for 1 to 2 months, followed by gradual tapering by 5-10 mg/mo until the maintenance dosage ( $5 \mathrm{mg} /$ day) was reached. After confirming clinical remission, the maintenance dosage was continued for an average of 6 months and then completely stopped. At the initial diagnosis, patients with obstructive jaundice underwent endoscopic retrograde cholangiopancreatography (ERCP) with endobiliary biopsy to perform biliary drainage for quicker resolution of symptoms and abnormal liver biochemistries, and to exclude possible cholangiocarcinoma before steroid treatment. In patients with relapse, corticosteroid therapy was re-administered according to the prior regimen.

\section{Statistical analysis}

Descriptive statistics are reported as frequency (percentage), mean \pm standard deviation (SD) or median (interquartile range, IQR) as appropriate. Differences in CT or MRCP findings between the AIP cohort and the control group were determined with the chi-square test or Fisher exact test. Stepwise multiple logistic regression analysis was performed to determine which imaging features were statistically significant in differentiating AIP from acute pancreatitis. All statistical analyses were performed using SPSS version 22 (IBM Corp., Armonk, NY, USA), with the results considered significant at $p<0.05$.

\section{RESULTS}

\section{Overall profile of all patients with type 2 AIP}

\section{1) Clinical findings}

The baseline characteristics of patients with type 2 AIP are summarized in Table 2. Among 244 patients with AIP, 183 (79.1\%) were diagnosed as having type 1 AIP and 27 (11.1\%) as having type 2 AIP. Twenty-four cases (9.8\%) of not diagnosed as type 1 and type 2 AIP patients were classified as AIP-NOS (Fig. 1). The median age of patients with type 2 AIP was 29 years (IQR, 20 to 39 years) and male was predominant (70.4\%). Of the 27 patients with type 2 AIP, 15 (55.6\%) were diagnosed as having definite AIP and 12 (44.5\%) was categorized as having probable AIP (Figs 2 and 3).

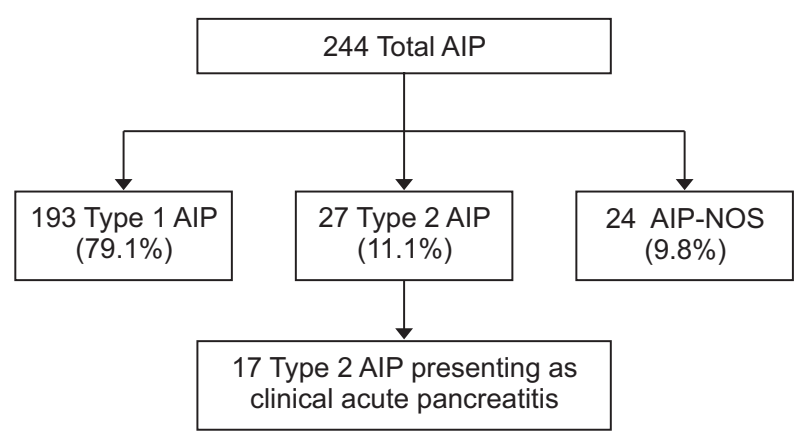

Fig. 1. Flowchart of patients with type 2 AIP presenting as clinical acute pancreatitis.

AIP, autoimmune pancreatitis; NOS, not otherwise specified.
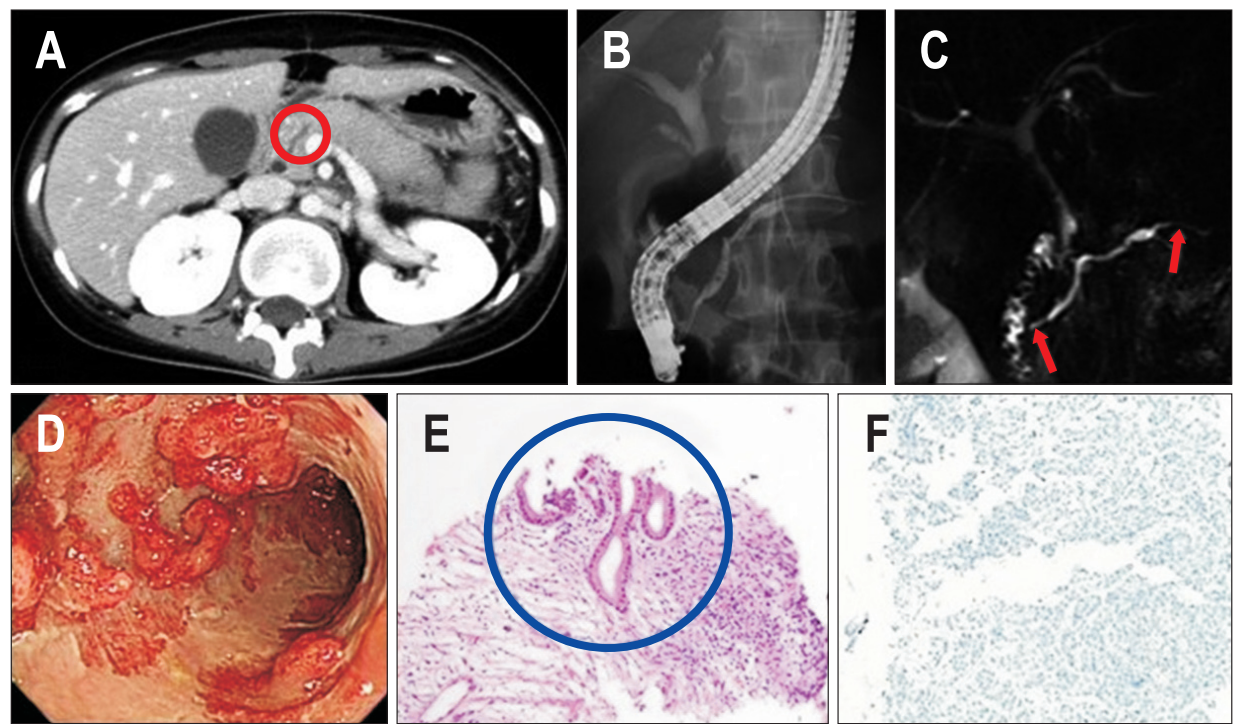

Fig. 2. A case of a 27-year-old female with definite type 2 autoimmune pancreatitis. (A) Computed tomography showing diffuse pancreas enlargement with focal main pancreatic duct dilatation (red circle). (B) Endoscopic retrograde pancreatography showing multifocal strictures of the main pancreatic duct. (C) Magnetic resonance cholangiopancreatography also revealing multifocal strictures (arrows) of the main pancreatic duct. (D) Colonoscopy showing friable, erythematous colonic mucosa with loss of normal vascular markings and inflammatory polyps, which are consistent with ulcerative colitis. (E) Pancreatic histology showing granulocytic epithelial lesions (H\&E, ×100). (F) Few immunoglobulin G4 (IgG4)-positive cells were identified on IgG4 immunostaining $(\times 100)$. 

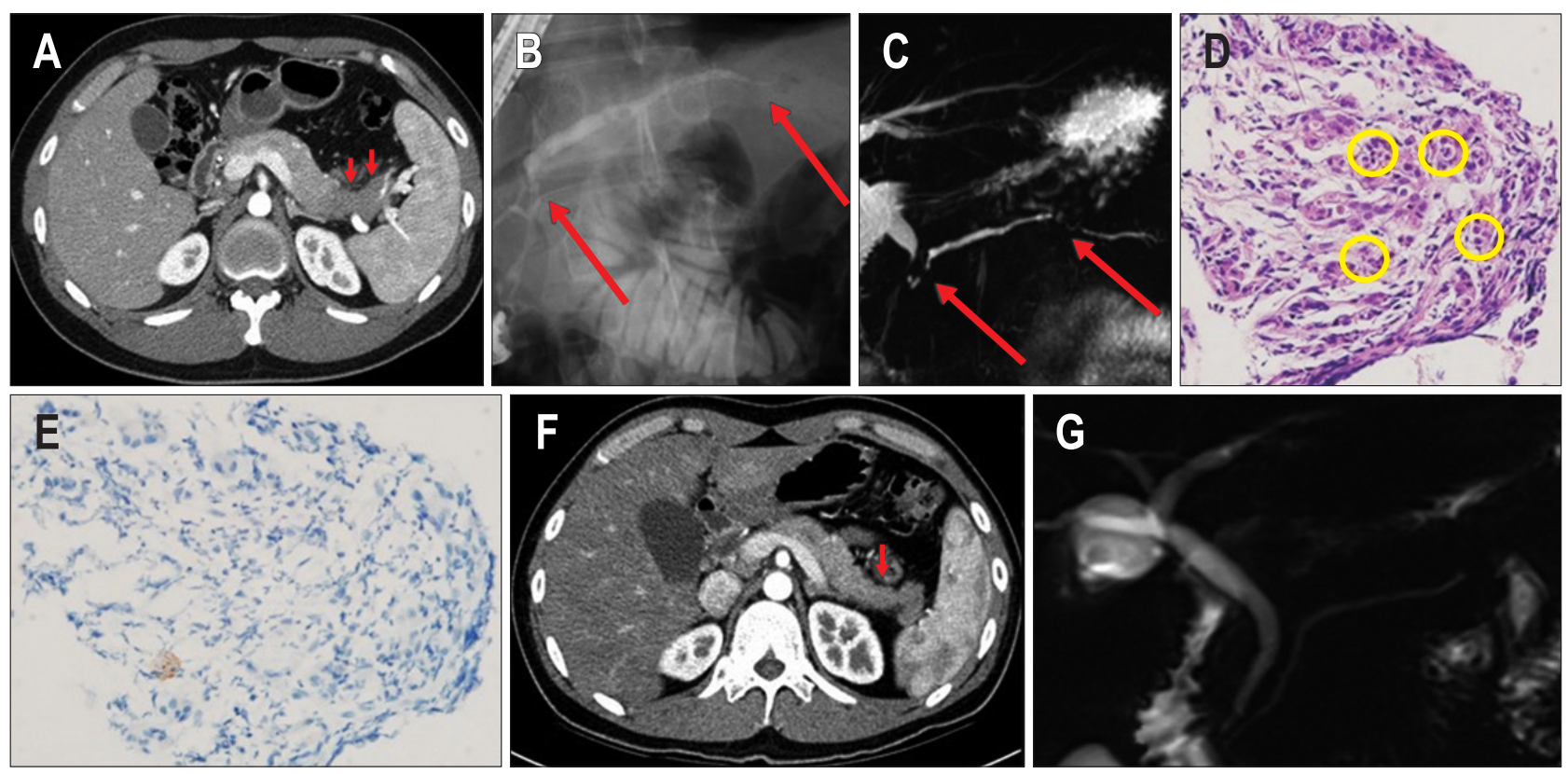

Fig. 3. A case of a 28-year-old male with probable type 2 autoimmune pancreatitis. (A) Computed tomography (CT) showing a low-density mass (arrows) at the pancreas tail. (B, C) Endoscopic retrograde pancreatography and magnetic resonance cholangiopancreatography (MRCP) revealing multifocal strictures (arrows) at the head \& tail portions of the main pancreatic duct. (D) Pancreatic histology revealing neutrophilic infiltration (circles) in acinar cells $(\mathrm{H} \& \mathrm{E}, \times 100)$. (E) Few immunoglobulin G4-positive cells are identified $(\times 100)$. (F, G) CT revealing the disappearance of the low-density mass (arrow) on the pancreas tail and MRCP showing the resolution of multifocal strictures of the main pancreatic duct after steroid administration.

Among patients with type 2 AIP, two patients (7.4\%, 2/27) showed elevation of serum IgG4 level but less than 2 times the upper limit of normal. Of them, one patient was diagnosed as having definite type 2 AIP with GEL. The other patient who did not undergo histopathological examination was diagnosed as having probable type 2 AIP with ulcerative colitis (UC).

Pancreatic tissue samples were obtained in 20 patients (74.1\%) including one from surgical resection, three from percutaneous transabdominal US-guided core biopsy, and 16 from EUS-PCB.

Twelve patients $(12 / 27,44.4 \%)$ had associated IBD (UC; 100\%); eight developed UC before the diagnosis of AIP and four (33.3\%) were diagnosed as having type 2 AIP and UC simultaneously.

\section{2) Treatment and relapse of patients with type 2 AIP}

Initial treatment consisted of steroid therapy in 26 patients (96.3\%) or surgical resection in one patient (3.7\%). All patients who received steroids without surgical resection showed complete clinical and radiological remission. During the median 32.3 months (IQR, 10.1 to 63 months) of follow-up, two of 25 patients (8\%) (two; follow-up loss) experienced one episode of disease relapse. One patient had relapse during steroid taper, and the other experienced relapse 10 months after steroid discontinuation. All patients with recurrence responded again to the reintroduction of steroids.

\section{Baseline characteristics of patients with type 2 AIP pre- senting as clinical acute pancreatitis}

The characteristics of patients with type 2 AIP who presented as clinical acute pancreatitis are summarized in Table 2. The median age was 29 years (IQR, 21 to 38 years) and patients were predominantly male (70.6\%). All patients required analgesics for pain control and 12 patients (70.6\%) visited the emergency department. Before the diagnosis of type 2 AIP presenting as clinical acute pancreatitis, nine patients (52.9\%) had medical history of idiopathic acute pancreatitis. All type 2 AIP patients presenting as acute pancreatitis had clinically mild pancreatitis. Among these patients with type 2 AIP presenting as clinical acute pancreatitis $(n=17)$, a total of five patients (29.4\%) were diagnosed as having definite AIP and 12 patients (76.5\%) as having probable AIP and UC was associated with eight patients (8/17, 47.1\%).

\section{Evaluation of radiological findings between type 2 AIP} presenting as clinical pancreatitis and ordinary acute interstitial edematous pancreatitis of gallstone etiology

Seventeen patients with type 2 AIP presenting as clinical pancreatitis and 51 patients with acute gallstone pancreatitis were compared. Among them, dynamic contrast-enhanced CT scans were obtained in 16 patients with type 2 AIP and in 29 patients with acute gallstone pancreatitis. The differences in the imaging 
Table 3. Comparison of Radiological Findings between Patients with Type 2 AIP Presenting as Acute Pancreatitis and Acute Gallstone Pancreatitis

\begin{tabular}{|c|c|c|c|}
\hline & $\begin{array}{l}\text { Type } 2 \text { AIP presenting as } \\
\text { acute pancreatitis }\end{array}$ & $\begin{array}{l}\text { Acute gallstone } \\
\text { pancreatitis }\end{array}$ & $\mathrm{p}$-value \\
\hline No. of CT findings of the pancreas & 17 & 51 & \\
\hline Multifocal lesion & $6(35.3)$ & 0 & $<0.01$ \\
\hline Focal mass & $9(52.9)$ & $2(3.9)$ & $<0.01$ \\
\hline Capsule-like low-density rim & $2(11.8)$ & 0 & 0.01 \\
\hline Delayed enhancement* & $13(81.3)$ & 0 & $<0.01$ \\
\hline Pancreas enlargement & $17(100)$ & $37(72.5)$ & 0.02 \\
\hline Peripancreatic fat infiltration & $10(58.8)$ & $46(90.2)$ & $<0.01$ \\
\hline Pancreatic fluid collection & $3(17.7)$ & $34(66.7)$ & $<0.01$ \\
\hline Focal/segmental MPD dilatation & $14(82.4)$ & $2(3.7)$ & $<0.01$ \\
\hline No. of MRCP findings & 16 & 27 & \\
\hline MPD narrowing & $16(100)$ & $2(7.4)$ & $<0.01$ \\
\hline MPD multifocal narrowing & $14(87.5)$ & 0 & $<0.01$ \\
\hline CBD narrowing & $4(25)$ & $1(3.7)$ & $<0.01$ \\
\hline
\end{tabular}

Data are presented as number (\%).

AIP, autoimmune pancreatitis; CT, computed tomography; MPD, main pancreatic duct; MRCP, magnetic resonance cholangiopancreatography; CBD, common bile duct.

*Dynamic contrast-enhanced CT was performed in 16 patients with AIP and in 29 patients with acute gallstone pancreatitis.
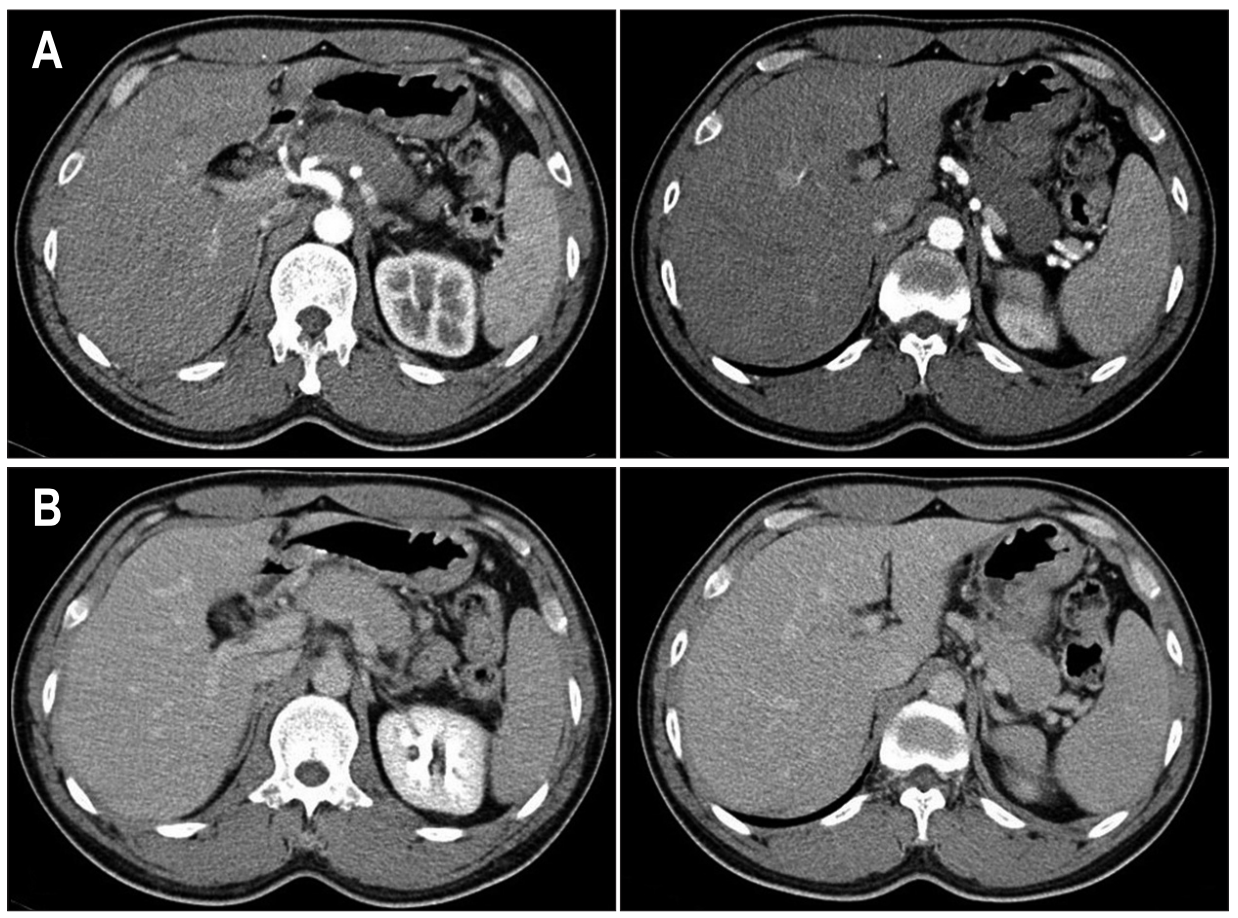

Fig. 4. Computed tomography imaging showing delayed enhancement of diffusely enlarged pancreas with loss of normal lobulated contour in one patient with type 2 autoimmune pancreatitis presenting as clinical acute pancreatitis. (A) Hypoattenuation of the enlarged pancreas (compared to the spleen) in the arterial phase. (B) Hyperattenuation of the enlarged pancreas (indicating delayed enhancement) in the portal venous phase.

findings between type 2 AIP and acute gallstone pancreatitis are summarized in Table 3.

Multifocality (35.3\% vs $0 \%, \mathrm{p}<0.01$ ), peripancreatic halo (11.8\% vs $0 \%, \mathrm{p}=0.01)$ and delayed enhancement (81.3\% vs $0 \%, p<0.01$ ) were only observed in type 2 AIP. Focal mass (52.9\% vs 3.9\%, $\mathrm{p}<0.01)$ was significantly more frequent in type
2 AIP than in acute gallstone pancreatitis. Peripancreatic fat infiltration (58.8\% vs 90.2\%, p<0.01) and peripancreatic fluid collection (17.7\% vs 66.7\%, $\mathrm{p}<0.01$ ) were significantly more frequent in acute gallstone pancreatitis than in type 2 AIP. Focal/segmental MPD dilatation was more frequently observed in type 2 AIP $(82.4 \%$ vs $3.7 \%, p<0.01)$. In terms of disease extent 
based on CT scans, the extent of pancreatic involvement was not statistically different between type 2 AIP (diffuse in 52.9\%, focal in 11.8\%, multifocal in 35.3\%) and acute gallstone pancreatitis (diffuse in 60.8\%, focal in 11.8\%, not definite in $27.5 \%$ ) $(\mathrm{p}=0.96)$.

MRCP was performed in 16 patients with AIP and 27 patients with acute gallstone pancreatitis. In the comparison of MRCP findings, presence of MPD narrowing (100\% vs 7.4\%, p<0.01), multifocal MPD narrowing (87.5\% vs $0 \%, \mathrm{p}<0.01)$, and CBD narrowing $(25 \%$ vs $3.7 \%, \mathrm{p}<0.01)$ were more frequently observed in type 2 AIP than in acute gallstone pancreatitis.

Of 17 patients, 13 (76.5\%) underwent endoscopic retrograde pancreatography (ERP); 11 (84.6\%) of patients showed a long ( $>1 / 3$ of the MPD length) or multifocal strictures without marked upstream dilatation (duct diameter $<5 \mathrm{~mm}$ ). Segmental/ focal narrowing without marked upstream dilatation was noted in two patients (15.4\%). Mild post-ERCP pancreatitis occurred in one patient (7.7\%), who was fully recovered after conservative treatment.

\section{DISCUSSION}

There is an ongoing debate whether type 1 AIP is more frequent in East Asia, whereas type 2 AIP prevails in Western countries. ${ }^{20}$ The relative proportion of type 2 AIP among total AIP is generally known to be higher in the West when compared to the East. In the literature, prevalence rates of type 2 AIP in the West vary widely (12.9\% to $45 \%) .^{21,22}$ In our study, the relative proportion of type 2 AIP in South Korea (11.1\%) was not as rare as previously thought. This might suggest that type 2 AIP has been overlooked for many years in East Asia since the histological diagnostic criteria of IDCP was originally established in the West based on histological findings of surgically resected pancreas specimens from patients with non-alcoholic mass-forming pancreatitis and Asian pathologists were not familiar with characteristic histology of IDCP. Different diagnostic approaches in East Asia may also contribute to the relatively lower prevalence rate of type 2 AIP. In South Korea and Japan, diagnostic ERP and a subsequent diagnostic steroid trial have been more actively used in patients with suspected AIP than in the West; type 2 AIP cases can therefore be mistakenly classified as simply AIP or AIP-NOS (in the absence of diagnostic histology) because pancreatic imaging findings are identical between type 1 and type 2 AIP and both subtypes well respond to steroids.

We compared CT findings between patients with type 2 AIP and those with acute interstitial edematous pancreatitis. In our study, patients with type 2 AIP had distinct CT features such as diffusely enlarged pancreas with capsule-like low density rim, a focal mass with homogenous enhancement, focal/segmental MPD dilatation without an obvious mass, absent/minimal peripancreatic fat infiltration or fluid collection of gallstone etiol- ogy, as compared to those with ordinary acute interstitial edematous pancreatitis (Table 3). On dynamic CT, most patients with a diffuse form of type 2 AIP revealed hypoattenuation of the enlarged pancreas in the arterial phase and iso- or hyperattenuation (compared to the spleen) in the portal venous phase indicating delayed enhancement (Fig. 4). In the focal form of type 2 AIP, delayed homogeneous enhancement of focal enlargement/ mass was seen. On the other hand, patients with gallstone pancreatitis demonstrated diffuse or localized enlargement of the pancreas and relatively normal enhancement of the pancreatic parenchyma with frequent peripancreatic fluid collection. In line with our results, recent studies showed that delayed enhancement and absent of peripancreatic stranding differentiated AIP from ordinary acute interstitial edematous pancreatitis. ${ }^{14,23} \mathrm{Com}-$ binations of pancreatic parenchymal morphology and enhancement pattern on CT and ductal imaging on MRCP/ERCP may give an initial clue to the suspicion of type 2 AIP. This has clinical implications since pancreatic biopsy is rarely performed in young patients with acute pancreatitis. It is therefore important to be familiar with the characteristic imaging findings of AIP. ${ }^{24}$ In our study, a relatively specific change of the MPD in type 2 AIP was skipped non-visualization of MPD indicating multifocal narrowing (Table 3$)^{24}$

Type 2 AIP may be the subgroup which potentially benefits the most from pancreatographic features (MRCP/ERCP) because the typical serological abnormalities and other organ involvement seen in type 1 AIP are not seen in type 2 AIP. ${ }^{5}$ At present, the necessity of diagnostic ERP to reliably assess pancreatic ductal morphology has been diminished by improvement in spatial resolution of MR pancreatography. In recent studies the MR pancreatography at 3.0 T or a 3-dimensional MR pancreatography with partial maximum intensity projection improved visualization of MPD and was comparable to ERP in terms of diagnostic accuracy in patients with AIP. ${ }^{25-27}$ This has clinical implications since many endoscopists avoid performing diagnostic ERP due to the fear of post-ERP pancreatitis in the setting of clinical acute pancreatitis and pancreatic biopsy is rarely performed in young patients with acute pancreatitis. ${ }^{26,27}$ In this study, MPD narrowing was present in all patients with type 2 AIP but was seen in only $7.4 \%$ of patients with gallstone pancreatitis. Particularly, multifocal MPD narrowing was observed in $87.5 \%$ of patients with type 2 AIP in contrast to none in patients with gallstone pancreatitis. If the pancreatogram reveals a diffuse/segmental or multifocal narrowing of the MPD on MRCP in a patient with apparently mild acute interstitial edematous pancreatitis on CT, this unusual association may suggest type 2 AIP presenting as acute pancreatitis rather than ordinary mild acute pancreatitis.

ICDC utilizes the co-occurrence of IBD as a supportive diagnostic criterion of type 2 AIP. In patients with type 2 AIP, association of IBD is common. In our study, nearly half (44.4\%) of type 2 AIP patients had associated IBD (100\% UC). In the 
literature, most cases with coexisting AIP and IBD were type 2 AIP. Also, in a Japanese study, IBD was seen in only type 2 AIP patients. ${ }^{6}$ A recent European multicenter study reported that the subtype of AIP in 91 individuals with coexisting AIP and IBD (58 UC and 33 Crohn's disease) was almost all type 2 AIP (89/91, type 2 AIP; $2 / 91$, type 1 AIP). ${ }^{28}$ On the other hand, the frequency of AIP in IBD patients is very low in South Korea and Japan; $0.4 \%$ each, respectively. ${ }^{17,29}$ Considering the low prevalence of type 2 AIP with IBD, it may be necessary to narrow down candidate IBD patients for diagnosing type 2 AIP.

It is clinically important to know when to look for type 2 AIP as a cause of acute pancreatitis since type 2 AIP is a rare disease and tissue acquisition is needed for a definitive diagnosis. In our study (Table 2), the most common clinical presentation of type 2 AIP was clinical acute pancreatitis (17/27, 63\%). Like ours, a European study showed that the most common clinical presentation was acute pancreatitis (80\%), followed by abdominal pain (11\%), and obstructive jaundice (7\%). ${ }^{28}$ And in a Japanese study, a characteristic feature of type 2 AIP was a significantly lower frequency of obstructive jaundice $(0 / 15,0 \%) .^{30}$ To diagnose type 2 AIP cases more, special attention should be paid to a specific subpopulation such as IBD patients who present as clinical acute pancreatitis. However, more common causes such as gallstones, alcohol and medication should first be excluded. In a recent study, among 58 patients with coexisting type 2 AIP and IBD who develop acute pancreatitis, the potential etiology of acute pancreatitis in 35 patients (60.3\%) were closely associated with immunosuppressive drugs used for IBD, such as mesalazine and azathioprine. ${ }^{28}$ Among patients with coexisting type 2 AIP and UC in the present study, type 2 AIP most occurred after the diagnosis of UC. And these patients were already taking medication for the management of UC at the time of AIP diagnosis. After these patients went to AIP remission with steroid therapy, they restarted azathioprine/mesalazine and none of them had azathioprine/mesalazine-induced pancreatic symptoms. Druginduced pancreatitis could be reliably excluded on the basis of negative rechallenge of a suspicious drug.

The relapse rate of type 2 AIP is generally considered to be significantly lower than in type 1 AIP. Due to the lower relapse rates in type 2 AIP, long-term maintenance therapy may not be necessary. In our study, the relapse rate of type 2 AIP was about $8 \%$. In a study from the Mayo Clinic, the cumulative relapse rate was $10.6 \%$ at 3 years (median follow-up, 2.9 years). ${ }^{3}$ In a recent European study, however, the relapse of type 2 AIP was $34 \%{ }^{28}$ The authors suggested that longer follow-up period ( $>5$ years) and fewer pancreatic surgery procedures was attributable to the high relapse rate. There is a possibility that higher relapse rate might be a consequence of contamination with type 1 AIP cases since even type 1 AIP patients have higher prevalence of UC than general population and level 2 supportive histology for type 2 AIP (acinar neutrophil infiltration) is also seen in type 1 AIP. $^{4,21}$

Our study has several limitations. First, this study was conducted by retrospective design and the number of cases is relatively small. However, AIP itself is a rare disease, and type 2 AIP is even rarer. Second, the prevalence rate of type 2 AIP in our study was from a single tertiary referral center with a specialized clinic that has been recognized for its experience and expertise with pancreatic diseases and may confer a considerable referral bias with an artificially increased frequency of type 2 AIP.

In conclusion, type 2 AIP is rare but clinically relevant in South Korea; the relative proportion of type 2 AIP among total AIP is $11.1 \%$. Gastroenterologists should have a high index of clinical suspicion for type 2 AIP in young IBD (specially UC) patients who present with clinical acute pancreatitis of uncertain etiology. Efforts to increase the amount of pancreatic tissue obtained by EUS-PCB are also required. As our knowledge and experience of type 2 AIP has accumulated, diagnostic ability will increase.

\section{CONFLICTS OF INTEREST}

No potential conflict of interest relevant to this article was reported.

\section{AUTHOR CONTRIBUTIONS}

Study concept and design: M.H.K. Acquisition of data analysis and interpretation of data: D.O., T.J.S., S.H.M., J.H.K., J.N.L., S.M.H., J.S.L., S.J.J., D.H.C., D.H.P., S.S.L., D.W.S., S.K.L., M.H.K. Drafting of the manuscript: D.O., T.J.S. Critical revision of the manuscript for important intellectual content: D.O., T.J.S., J.H.K., S.M.H., J.N.L., J.S.L., S.J.J., S.M.H. Statistical analysis: D.O., T.J.S. Supervision: M.H.K.

\section{ORCID}

Dongwook Oh

Tae Jun Song Sung-Hoon Moon Jin Hee Kim

Nam Joo Lee Seung-Mo Hong Joune Seup Lee Seok Jung Jo Dong Hui Cho Do Hyun Park Sang Soo Lee Dong-Wan Seo Sung Koo Lee Myung-Hwan Kim https://orcid.org/0000-0002-6084-0138 https://orcid.org/0000-0002-6156-8746 https://orcid.org/0000-0002-7879-3114 https://orcid.org/0000-0001-5036-3326 https://orcid.org/0000-0001-8751-129X https://orcid.org/0000-0002-8888-6007 https://orcid.org/0000-0003-4186-9629 https://orcid.org/0000-0002-5695-9849 https://orcid.org/0000-0001-9003-9184 https://orcid.org/0000-0002-0270-6969 https://orcid.org/0000-0002-3785-2341 https://orcid.org/0000-0001-7525-8151 https://orcid.org/0000-0003-4155-4416 https://orcid.org/0000-0003-0992-6734 


\section{REFERENCES}

1. Madhani K, Farrell JJ. Autoimmune pancreatitis: an update on diagnosis and management. Gastroenterol Clin North Am 2016;45:29-43.

2. Song TJ, Kim MH, Kim MJ, Moon SH, Han JM; International Panel of Speakers and Moderators. Clinical validation of the International Consensus Diagnostic Criteria and algorithms for autoimmune pancreatitis: combined IAP and KPBA meeting 2013 report. Pancreatology 2014;14:233-237.

3. Hart PA, Levy MJ, Smyrk TC, et al. Clinical profiles and outcomes in idiopathic duct-centric chronic pancreatitis (type 2 autoimmune pancreatitis): the Mayo Clinic experience. Gut 2016;65:17021709.

4. Kamisawa T, Chari ST, Giday SA, et al. Clinical profile of autoimmune pancreatitis and its histological subtypes: an international multicenter survey. Pancreas 2011;40:809-814.

5. Song TJ, Kim JH, Kim MH, et al. Comparison of clinical findings between histologically confirmed type 1 and type 2 autoimmune pancreatitis. J Gastroenterol Hepatol 2012;27:700-708.

6. Notohara K, Nishimori I, Mizuno N, et al. Clinicopathological features of type 2 autoimmune pancreatitis in Japan: results of a multicenter survey. Pancreas 2015;44:1072-1077.

7. Kamisawa T, Chari ST, Lerch MM, Kim MH, Gress TM, Shimosegawa T. Recent advances in autoimmune pancreatitis: type 1 and type 2. Gut 2013;62:1373-1380.

8. Shimosegawa T, Chari ST, Frulloni L, et al. International Consensus Diagnostic Criteria for autoimmune pancreatitis: guidelines of the International Association of Pancreatology. Pancreas 2011;40:352-358.

9. Chari ST, Smyrk TC, Levy MJ, et al. Diagnosis of autoimmune pancreatitis: the Mayo Clinic experience. Clin Gastroenterol Hepatol 2006;4:1010-1016.

10. Otsuki M, Chung JB, Okazaki K, et al. Asian diagnostic criteria for autoimmune pancreatitis: consensus of the Japan-Korea Symposium on Autoimmune Pancreatitis. J Gastroenterol 2008;43:403408.

11. Tenner S, Baillie J, DeWitt J, Vege SS; American College of Gastroenterology. American College of Gastroenterology guideline: management of acute pancreatitis. Am J Gastroenterol 2013;108:1400-1415.

12. Forsmark CE, Baillie J; AGA Institute Clinical Practice and Economics Committee; AGA Institute Governing Board. AGA Institute technical review on acute pancreatitis. Gastroenterology 2007;132:2022-2044.

13. Frulloni L, Scattolini C, Falconi M, et al. Autoimmune pancreatitis: differences between the focal and diffuse forms in 87 patients. Am J Gastroenterol 2009;104:2288-2294.

14. Lee-Felker SA, Felker ER, Kadell B, et al. Use of MDCT to differentiate autoimmune pancreatitis from ductal adenocarcinoma and interstitial pancreatitis. AJR Am J Roentgenol 2015;205:2-9.

15. Takahashi N, Fletcher JG, Hough DM, et al. Autoimmune pancre- atitis: differentiation from pancreatic carcinoma and normal pancreas on the basis of enhancement characteristics at dual-phase CT. AJR Am J Roentgenol 2009;193:479-484.

16. Edge MD, Hoteit M, Patel AP, Wang X, Baumgarten DA, Cai Q. Clinical significance of main pancreatic duct dilation on computed tomography: single and double duct dilation. World J Gastroenterol 2007;13:1701-1705.

17. Kim JW, Hwang SW, Park SH, et al. Clinical course of ulcerative colitis patients who develop acute pancreatitis. World J Gastroenterol 2017;23:3505-3512.

18. Moon SH, Kim MH, Park DH, et al. Is a 2-week steroid trial after initial negative investigation for malignancy useful in differentiating autoimmune pancreatitis from pancreatic cancer? A prospective outcome study. Gut 2008;57:1704-1712.

19. Maire F, Le Baleur Y, Rebours V, et al. Outcome of patients with type 1 or 2 autoimmune pancreatitis. Am J Gastroenterol 2011;106:151-156.

20. Petzold G, Ellenrieder V, Neesse A. Autoimmune pancreatitis in Germany: rare but relevant. Digestion 2017;96:185-186.

21. Sah RP, Chari ST, Pannala R, et al. Differences in clinical profile and relapse rate of type 1 versus type 2 autoimmune pancreatitis. Gastroenterology 2010;139:140-148.

22. Hart PA, Kamisawa T, Brugge WR, et al. Long-term outcomes of autoimmune pancreatitis: a multicentre, international analysis. Gut 2013;62:1771-1776.

23. Graziani R, Frulloni L, Mantovani W, et al. Autoimmune pancreatitis and non-necrotizing acute pancreatitis: computed tomography pattern. Dig Liver Dis 2012;44:759-766.

24. Takahashi N. CT and MR features of autoimmune pancreatitis [Internet]. Pancreapedia: Exocrine Pancreas Knowledge Base c2013 [cited 2019 Mar 7]. Available from https://doi.org/10.3998/ panc.2013.15.

25. Park SH, Kim MH, Kim SY, et al. Magnetic resonance cholangiopancreatography for the diagnostic evaluation of autoimmune pancreatitis. Pancreas 2010;39:1191-1198.

26. Kim JH, Byun JH, Kim MH, et al. Pancreatic duct in autoimmune pancreatitis: intraindividual comparison of magnetic resonance pancreatography at 1.5 T and 3.0 T. Pancreas 2017;46:921-926.

27. Yanagisawa S, Fujinaga Y, Watanabe T, et al. Usefulness of threedimensional magnetic resonance cholangiopancreatography with partial maximum intensity projection for diagnosing autoimmune pancreatitis. Pancreatology 2017;17:567-571.

28. Lorenzo D, Maire F, Stefanescu C, et al. Features of autoimmune pancreatitis associated with inflammatory bowel diseases. Clin Gastroenterol Hepatol 2018;16:59-67.

29. Ueki T, Kawamoto K, Otsuka Y, et al. Prevalence and clinicopathological features of autoimmune pancreatitis in Japanese patients with inflammatory bowel disease. Pancreas 2015;44:434-440.

30. Kawa S, Okazaki K, Notohara K, Watanabe M, Shimosegawa T; Study Group for Pancreatitis Complicated with Inflammatory Bowel Disease organized by The Research Committee for Intractable Pancreatic Disease (Chairman: Tooru Shimosegawa) and The 
470 Gut and Liver, Vol. 13, No. 4, July 2019

Research Committee for Intractable Inflammatory Bowel Disease (Chairman: Mamoru Watanabe), both of which are supported by the Ministry of Health, Labour, and Welfare of Japan. Autoim- mune pancreatitis complicated with inflammatory bowel disease and comparative study of type 1 and type 2 autoimmune pancreatitis. J Gastroenterol 2015;50:805-815. 